\title{
The Individual Recovery Outcomes Counter: preliminary validation of a personal recovery measure
}

\author{
Bridey Monger, ${ }^{1,2}$ Scott M. Hardie, ${ }^{1}$ Robin Ion, ${ }^{1}$ Jane Cumming, ${ }^{2}$ Nigel Henderson ${ }^{2}$
}

The Psychiatrist (2013), 37, 221-227, doi: 10.1192/pb.bp.112.041889

${ }^{1}$ University of Abertay Dundee, UK; ${ }^{2}$ Penumbra, Edinburgh, UK

Correspondence to Scott M. Hardie (s.hardie@abertay.ac.uk)

First received 30 Oct 2012, final revision 13 Feb 2013, accepted 26 Feb 2013

\begin{abstract}
Aims and method The Individual Recovery Outcomes Counter (I.ROC) is to date the only recovery outcomes instrument developed in Scotland. This paper describes the steps taken to initially assess its validity and reliability, including factorial analysis, internal consistency and a correlation benchmarking analysis.
\end{abstract}

Results The I.ROC tool showed high internal consistency. Exploratory factor analysis indicated a two-factor structure comprising intrapersonal recovery (factor 1) and interpersonal recovery (factor 2 ), explaining between them over $50 \%$ of the variance in I.ROC scores. There were no redundant items and all loaded on at least one of the factors. The I.ROC significantly correlated with widely used existing instruments assessing both personal recovery and clinical outcomes.

Clinical implications I.ROC is a valid and reliable measure of recovery in mental health, preferred by service users when compared with well-established instruments. It could be used in clinical settings to map individual recovery, providing feedback for service users and helping to assess service outcomes.

Declaration of interest B.M., N.H. and J.C. are currently employed by Penumbra. R.I. and S.H. are currently employed by the University of Abertay and B.M. was employed by Abertay during the research.
Since Anthony's seminal paper in $1993,{ }^{1}$ the recovery concept has become embedded in local and national mental health policy across the UK. Its advance into the mainstream can be gauged not just by the volume of academic literature devoted to it, but also by the scope and range of this work. In a recent literature search, we identified over 300 recovery-focused publications in the past 12 years. These ranged from personal accounts of the recovery journey ${ }^{2}$ to attempts to define or measure the concept, ${ }^{3,4}$ along with histories, ${ }^{5}$ empirical studies ${ }^{6,7}$ and signs of an emerging critique. ${ }^{8}$ Despite the large amount of research and the diversity within it, there are some emergent themes. Recovery is both an outcome and a process whereby 'recovery from' and 'recovery in' mental illness are fluid concepts that are not mutually exclusive. ${ }^{9}$ Recovery is subjective, explaining some of the difficulty in accurately defining it. ${ }^{10}$

\section{Measuring recovery}

To measure recovery, there needs to be agreement on the most common themes emerging from research. Davidson and colleagues $^{11}$ neatly summarise these as involving: (1) recovery as a journey; (2) being supported by others; (3) renewing hope and commitment; (4) engaging in meaningful activities; (5) redefining self; (6) incorporating illness; (7) overcoming stigma; (8) assuming control; (9) managing symptoms; (10) becoming empowered and exercising citizenship. This view is also supported by literature reviews examining recovery in Britain. ${ }^{12-14}$ These concur with the findings of our literature search as they show a similarity in the breadth of work and the key themes emerging from it.

Of particular significance to the present study is work that deals with the measurement of recovery. Effective measurement can provide valuable feedback to service users and mental health workers in terms of individual progress. It can also be used to help shape care planning and can be drawn on as evidence of outcomes by commissioners and managers within services. ${ }^{15}$

To date a range of tools have been developed to measure different aspects of recovery. These were reviewed by Burgess and colleagues. ${ }^{16}$ Where measures have focused on individual recovery and/or outcomes, as opposed to service orientation or practitioner attitudes, they can be criticised for an over-reliance on purely clinician-generated items (e.g. the Milestones of Recovery Scale, MORS); ${ }^{17}$ for their length (e.g. the Recovery Measurement Tool: 91 questions), ${ }^{18}$ which it is felt makes them inappropriate for routine use; ${ }^{16}$ and for their focus on symptom reduction as 
opposed to attributes of personal recovery ${ }^{4}$ (e.g. the Illness Management and Recovery (IMR) Scales). ${ }^{19}$ Moreover, given the subjectivity of the recovery concept, it has been argued that many of the tools currently available lack sensitivity to the needs of local populations. ${ }^{20}$ Specifically, the majority have been developed in North America and Australia and their relevance to the local, in this case UK, population is therefore unclear.

\section{Recovery Star}

One of the widely used tools in assessing recovery in the UK is the Recovery Star. ${ }^{21}$ It is a measure based on a 10-stage model of recovery where service users are asked to identify their current stage of recovery against ten indicators. Its psychometric properties have recently been analysed and high internal consistency and good test-retest reliability were reported. ${ }^{22,23}$ However, although the tool has demonstrated good convergent validity with a measure of social functioning, it did not correlate significantly with the Mental Health Recovery Measure. ${ }^{23}$ The authors conclude that it may therefore not be accurate to describe the Recovery Star as a measure of personal recovery. ${ }^{23}$ These data were published after the current study was undertaken, and although the initial results are promising, there is still a relative lack of detailed information on this tool.

\section{Process of Recovery Questionnaire}

Another popular UK recovery measurement tool is the Process of Recovery Questionnaire (QPR), a 22-item questionnaire measuring personal recovery on a 5-point Likert scale ranging from 'disagree strongly' to 'agree strongly'. Good internal consistency (for the two subscales identified), test-retest reliability and convergent validity have been reported with a small number of measures of aspects of recovery within a population with a history of psychosis. ${ }^{4}$ This has not yet been tested against any other measures of recovery or within a wider population of people with mental health problems, so full validity is yet to be established.

In summary, although two measures of recovery have been developed for use with UK populations, as yet neither of these has been subject to full, standardised psychometric testing. Also, their application may be limited as they are not necessarily focused towards personal recovery across all client groups (unlike I.ROC which was specifically developed to fulfil this role).

\section{Development of I.ROC}

The Individual Recovery Outcomes Counter (I.ROC) was developed by Scottish mental health charity Penumbra in 2007 to measure service users' 'distance travelled'. A working group of senior managers was established to investigate potential indicators felt to be pertinent to well-being and recovery. Drawing from experience, output from UK health and social care agencies (e.g. Health Scotland), and examining existing tools (including the Outcomes $\left.\operatorname{Star}^{24}\right),{ }^{21}$ led to the identification of 12 indicators relevant to Penumbra's work. An initial version of the I.ROC questionnaire was composed and subsequently refined based on feedback from a pilot group of 40 service users. After the refinement of the scoring and wording of questions, I.ROC was rolled out across Penumbra's services. Since 2011, Penumbra has been working with the University of Abertay Dundee to explore the psychometric properties of this tool. Focus groups with service users and staff identified more areas for improvement, resulting in changes to the wording and layout of the questionnaire. These changes were then confirmed with more focus groups and staff working groups to establish good content validity. ${ }^{25}$

\section{Method}

\section{Measures}

Comparative validity of I.ROC was assessed by asking participants to complete it along with two well-established outcome/recovery measurement questionnaires, the Recovery Scale (RAS) ${ }^{26,27}$ and the Behaviour and Symptom Identification Scale (BASIS-32). ${ }^{28,29}$ These tools were chosen because of their robust and widespread use within recovery and outcome measurement, both in practice and in the validation of other measures. Like I.ROC, both tools use a Likert scale, making answers easily comparable.

The revised I.ROC is a facilitated self-assessment, which is administered on a quarterly basis as part of service users' ongoing support. It consists of 12 questions, based around 12 indicators of recovery (Table 1).

The RAS is a 41-item questionnaire, scored on a 5-step scale of 'strongly disagree' to 'strongly agree'. It has been tested against other measures of recovery and has been shown to be both valid and reliable. ${ }^{30}$ It has demonstrated acceptable test-retest reliability $(r=0.88)$, good internal consistency $(\alpha=0.93)$ and convergent validity with measures of empowerment, self-esteem, social support and quality of life and hope. ${ }^{27,31}$ Convergent validity has also been established with other recovery measures including the Mental Health Recovery Measure, ${ }^{32}$ Stages of Recovery Instrument (STORI) ${ }^{33,34}$ and QPR. ${ }^{4}$ This makes it appropriate to use as a benchmark for personal recovery.

The second comparative tool, BASIS- $32^{28,29}$ is a 32 -item routine outcome measurement self-report questionnaire designed to measure clinical outcomes of interventions from the service user's perspective. It is widely applied in Australia and New Zealand, where national and state funders require services to collect and use outcome data. ${ }^{35}$ The tool has good test-retest reliability and internal consistency both overall (0.89) and for the identified subscales (0.65-0.81). ${ }^{36}$ It shows sensitivity to changes in functioning and symptoms ${ }^{37}$ and has been used as a comparative measure in the validation of recovery and outcomes measures, ${ }^{38,39}$ for example, in validating the Japanese version of the RAS, where it significantly negatively correlated with the RAS. $^{40}$ It has also been used in the assessment of recovery and rehabilitation-based interventions, treatments and programmes. ${ }^{41-43}$ As a clinical outcomes measure, BASIS-32 can be used to establish the validity of I.ROC as an outcome measure more broadly in line with routine outcome measurement.

\section{Participants}

Participants were all those currently receiving support in the community from Penumbra. There were no exclusion 


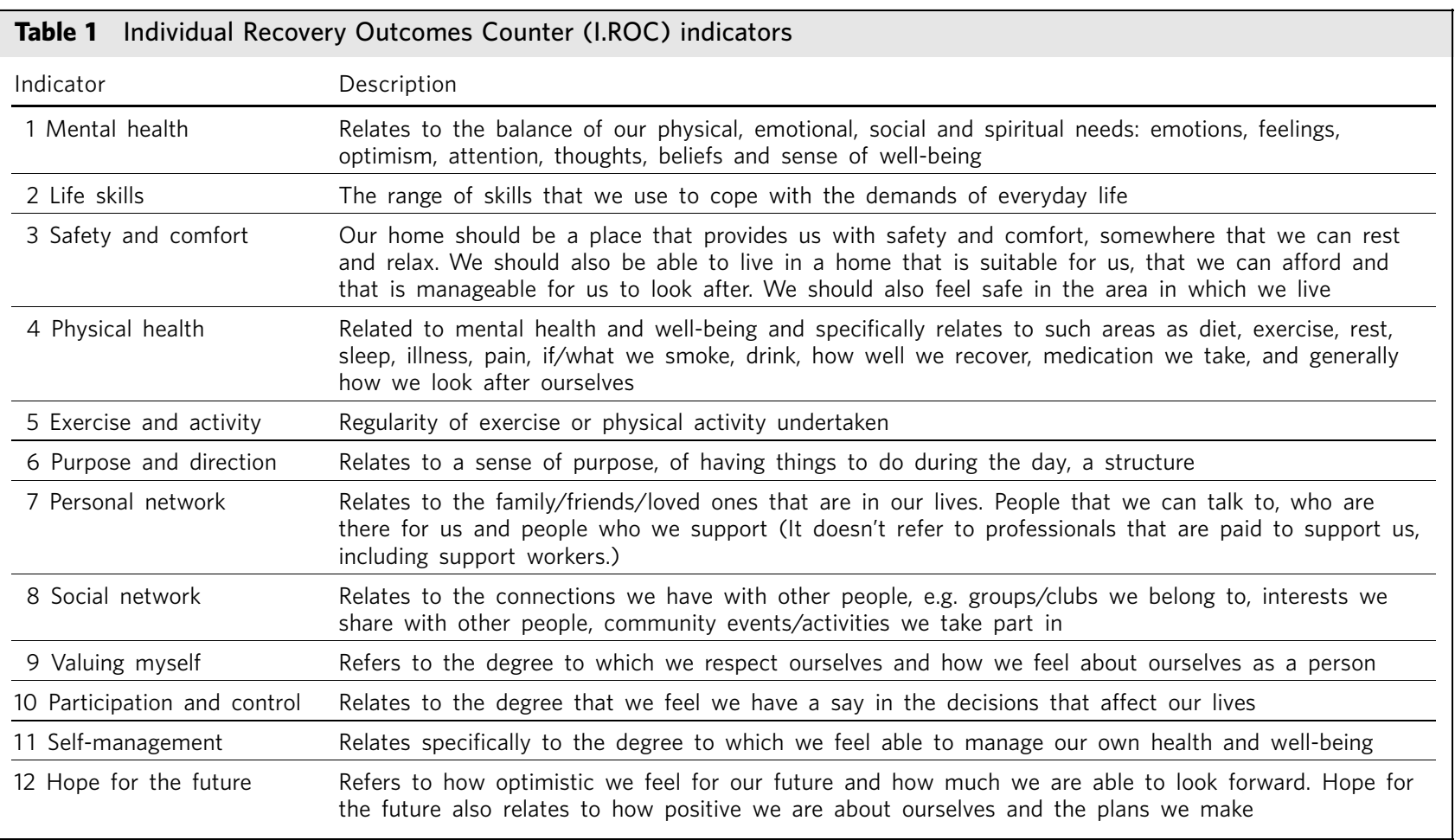

criteria, and data collection was carried out by Penumbra staff $(n=17)$, all of whom received training from the research team prior to the commencement of the study. Ethical approval was granted by the University of Abertay Ethics Committee. Participants were 79 women and 92 men ranging in age from 15 to 79 years, with a mean age of 46 years. One participant was excluded from analysis as they did not complete all three questionnaires. Mental illness diagnoses were largely self-reported and ranged from anxiety through to multiple, complex diagnoses. Between the 170 participants included in the analysis, there were 320 reported diagnoses, with the most common being depression, reported in over $50 \%$ of participants; 94 participants reported 2 or more diagnoses, with anxiety and depression the most common dual diagnosis. Participants largely lived alone $(66 \%)$ in rented or supported accommodation; $65 \%$ were unemployed, with only 10 participants in paid employment and 7 in education. Participants had been receiving support from Penumbra for varying lengths of time, ranging from 49 days to 20 years, with $70 \%$ receiving support for between 6 months and 2 years and $32 \%$ in their first year of service. This support ranged from occasional respite care through to 24-hour supported accommodation. Participants varied in the number of previous I.ROCs they have completed; an I.ROC is completed as soon as possible following intake and is then repeated every 3 months. The 55 individuals in their first year (32\%) had completed fewer than three I.ROCs, whereas $70 \%$ (120 individuals) had been in service less than 2 years completing seven or fewer I.ROCs.

\section{Procedure}

From November 2011 to April 2012, participants were asked to complete I.ROC, RAS and BASIS-32 with a support worker. Testing took place at a location of the participant's choosing, under Penumbra's lone working policy. After filling in a demographics questionnaire, participants completed the questionnaires (in a standardised format and counterbalanced order, with a third of participants filling in I.ROC as the first, as the second and as the third questionnaire) with the testers who read out each question to the participant before recording the answer. After finishing the final questionnaire, participants were asked to fill in a feedback form, briefly describing how they found the questionnaires and the testing experience.

\section{Analysis}

Quantitative methods were used to analyse the comparative validity and internal consistency of the questionnaire. Data were analysed with SPSS-19 for Windows. Analysis methods were similar to those used in other measurement tool validations. $^{31,33,44}$

\section{Results}

\section{Score distributions}

All three questionnaires were tested using the KolmogorovSmirnov test for normality, which showed that both I.ROC and BASIS-32 were normally distributed $\left(D_{170}=0.129\right.$, $P=0.200$ ), but RAS was significantly non-normal $\left(D_{170}=0.073, \quad P<0.05\right)$. Therefore comparisons between measures were carried out with non-parametric tests.

\section{Demographic/confounding variables}

The results of a Kruskal-Wallis (non-parametric ANOVA equivalent) determined that age was not a significant confounding variable for any measure (Table 2). 
Table 2 Examination of age and gender influence on scores $(n=170)$

\begin{tabular}{|c|c|c|c|c|}
\hline & \multicolumn{2}{|c|}{ Age (d.f. =6) } & \multicolumn{2}{|c|}{ Gender (d.f. = 1) } \\
\hline & $\chi^{2}$ & $P$ & $\chi^{2}$ & $P$ \\
\hline I.ROC & 2.77 & 0.837 & 0.017 & 0.896 \\
\hline BASIS-32 & 9.61 & 0.142 & 4.18 & $0.041^{\circ}$ \\
\hline RAS & 7.21 & 0.302 & 5.58 & $0.018^{\circ}$ \\
\hline
\end{tabular}

BASIS-32, Behaviour and Symptom Identification Scale; I.ROC, Individual Recovery Outcomes Counter; RAS, Recovery Scale. a. Denotes significance $(P<0.05)$

Scores on I.ROC did not differ significantly based on gender. This finding is in line with validations of RAS, ${ }^{30}$ STORI $^{33}$ and the Stages of Recovery Scale, ${ }^{38}$ all of which reported no significant differences dependent on gender. A different pattern of results was found on the other two scales, however. Unlike the original validation, males were found to score significantly higher on RAS and significantly lower on BASIS-32 than females. This suggests that men in the current sample were more likely to report higher recovery scores when using these questionnaires.

\section{Concurrent validity}

Scoring on I.ROC and RAS is similar, with higher scores indicating better well-being, whereas BASIS-32 uses an inverse scoring system with lower scores indicating better mental health. To measure the strength of the relationships between the questionnaires, Spearman's correlations (two-tailed) were calculated for the total scores on all three measures. Thus, I.ROC scores were significantly positively correlated to RAS scores $\left(r_{\mathrm{s}}=0.723, P<0.001\right)$ and significantly negatively correlated to BASIS-32 scores $\left(r_{\mathrm{s}}=-0.602, P<0.001\right)$. These results meet with a minimal criterion for correlation between similar psychometric instruments (0.55). ${ }^{45}$ We contend that this indicates positive initial support for the concurrent validity of I.ROC, demonstrating an ability to measure recoveryfocused outcomes in a way that is similar to the current leading measures.

Pearson's correlations were also calculated for subscales within BASIS-32 and RAS, along with the two I.ROC factors found during factor analysis. Both I.ROC factors were found to correlate significantly with the subscales on the other two measures. The only subscale correlation not to produce a significant result was between I.ROC factor 2 and the BASIS-32 psychosis subscale. This suggests that I.ROC compares favourably with the other two measures at a structural as well as general level.

\section{Internal consistency}

Internal consistency correlations were calculated using Cronbach's alpha. This is a measure of the relatedness of all questions within a questionnaire to each other and to a single overarching construct (in this case recovery). High scores indicate a strong relationship between all questions, thus determining the level of homogeneity of the tool. ${ }^{46}$ All three questionnaires have good internal consistency, although that of RAS was highest (0.96), closely followed by BASIS-32 (0.95). However, I.ROC produced a sufficiently high score (0.86) to indicate good internal consistency. ${ }^{47} \mathrm{It}$ should be noted, however, that both BASIS-32 and RAS consist of far more questions than I.ROC, which may have positively affected their score. The literature suggests that 0.8 is a good goal to aim for, and clearly I.ROC exceeds this. $^{48}$

\section{Factor analysis}

Exploratory factor analysis was performed to investigate I.ROC's underlying structure (Table 3). By investigating the correlations between each item on a questionnaire, it is possible to identify existing question groupings, which can be compared with the theories underpinning the measure.

\begin{tabular}{|c|c|c|c|}
\hline Intrapersonal (self-reflection/change) & Factor 1 loading & Interpersonal (outward/forward-looking) & Factor 2 loading \\
\hline 1 Mental health & 0.53 & 5 Exercise and activity & 0.71 \\
\hline 2 Life skills & 0.67 & 6 Purpose and direction & 0.71 \\
\hline 3 Safety and comfort & 0.66 & 8 Social network & 0.69 \\
\hline 4 Physical health & 0.48 & & \\
\hline 7 Personal network & 0.58 & 12 Hope for the future & 0.62 \\
\hline 9 Valuing myself & 0.71 & & \\
\hline 10 Participation and control & 0.65 & & \\
\hline 11 Self-management & 0.82 & & \\
\hline
\end{tabular}

I.ROC, Individual Recovery Outcomes Counter. 
In I.ROC, two significant factors were found using a varimax rotation.

The KMO statistic $^{49}$ was 'meritorious' (0.859). ${ }^{50}$ Bartlett's test of sphericity was highly significant, proving the correlation matrix not to be an identity matrix $(P<0.001)$, and the determinant of the correlation matrix $(\mathrm{d}=2.01, \mathrm{E}=0.007)$ indicated that principal components analysis was appropriate.

Exploratory factor analysis indicated that two underlying factors comprising eight and four items accounted for $51.8 \%$ of the variance in scores. Two items ('hope for the future' and 'physical health') loaded on both factors, so were assigned to the factor with a slightly higher loading (physical health: factor 1, 0.48; hope: factor 2, 0.62). Eigen values showed that factor 1 explained $40.7 \%$ of the variance in the data and factor 2 explained $11.1 \%$. Item loadings in the two extracted factors (highest factor loading) all exceeded 0.45. Internal consistency was good for factor 1 (Cronbach's $\alpha=0.83$ ) and acceptable for factor 2 (Cronbach's $\alpha=0.74$ ). ${ }^{32}$

The authors (academic researchers and mental health professionals) agreed that the factors identified by the analyses should be labelled as 'intrapersonal' and 'interpersonal'. These closely resemble factors in the QPR suggested by Neil and colleagues, ${ }^{4}$ where intrapersonal elements are described as 'tasks that the individual is responsible for conducting, and which help them to rebuild their lives'. These could include taking responsibility for the management of their physical and mental health and day-to-day life skills (e.g. cooking, cleaning) and for participating in choices that affect their lives. These types of behaviours have been described by Andresen et $a l^{51}$ as including 'self-determination and resilience'. Interpersonal items relate to reflection on the individual's value to external processes and relationships. These could include their ability to participate in social activities and to feel that they play a meaningful part in their own lives and their wider community. As in the QPR, of the two factors identified in I.ROC, the majority of items loaded on the intrapersonal subscale (17 items $v .5$ in QPR).

\section{Questionnaire preference}

After completing the three questionnaires, participants were asked to identify which questionnaire was their favourite and which one they liked least. Of the 124 service users who answered that question, $52 \%(n=64)$ chose I.ROC as their favourite. Significantly more participants selected I.ROC than either RAS $(n=35, t=5.996, P<0.001)$ or BASIS$32(n=26, t=7.245, P<0.001)$ as their favourite.

Conversely, BASIS-32 $(n=45)$ was found to be the least popular questionnaire, with significantly more participants selecting it as their least favourite than either I.ROC $(n=28$, $t=-4.49, P<0.001)$ or RAS $(n=30, t=-4.173, P<0.001)$. Approximately $50 \%$ or more of participants with a diagnosis of schizophrenia (60\%: $F=4.0$, d.f. $=2,42, P<0.05)$, anxiety (52\%) or depression (49\%) who selected a favourite questionnaire chose I.ROC. This trend was repeated irrespective of number of previous I.ROCs completed (percentage of participants who preferred I.ROCs: 0 previous I.ROCs, $40 \%$; 3 previous I.ROCs, $60 \%$; 7+ previous I.ROCs, $74 \%$ ). Participants who had been with Penumbra less than 6 months (57\%) preferred I.ROC, although not to a statistically significant degree (ANOVA (Welch): $F=3.25$, d.f. $=2,39, P=0.59$ ) as did those who had been with the organisation for 6 years (49-72 months: 58\%; $F=3.808$, d.f. $=2,33, \quad P<0.05)$. Questionnaire preference did not appear to be moderated by participants' scores on I.ROC. Those with scores between 37 and 72 (highest possible score) still significantly preferred I.ROC over RAS and BASIS-32 (score 37-48: $F=7.92, P<0.01 ; 49-60$ : $F=3.27$, $P<0.05 ; 71-72, F=4.52, P<0.05)$. Participants with low I.ROC scores still preferred I.ROC $(F=3.27, P=0.57)$, although not significantly.

\section{Discussion}

The evidence presented here supports the hypothesis that I.ROC is a valid and reliable recovery outcomes measure which can be used in routine clinical practice as a means of tracking service user progress, as an aid to care planning and as a means of assessing the impact of service inputs. It is significantly correlated with BASIS-32 and RAS, and the correlation between I.ROC and BASIS-32 increases the breadth of possible applications of I.ROC in the future, for example as an outcomes tool within public health and social care services. The internal consistency of I.ROC was high, with no item redundancy, suggesting that all questions contribute to a single recovery-related construct. This supports the content validity of the measure. Exploratory factor analysis revealed that this construct comprised two factors, labelled intrapersonal and interpersonal recovery, although further work is needed to understand their wider relationship to both the overall construct and other aspects of recovery.

On the whole, participants preferred I.ROC to the other measures. They described it as easy to complete and agreed that it helped them think about their recovery. It thus seems fair to conclude that I.ROC measures similar recovery and personal outcomes constructs to RAS and BASIS-32, but in a way that most service users found preferable. This was a consistent finding irrespective of demographic variables and test scores. Trends indicated that I.ROC total score was not a significant preference moderator, suggesting that participants feel comfortable with the measure no matter the current state of their mental health. Controlled empirical testing has supported the internal structure and validity of the tool. Qualitative analysis of service user focus groups and the feedback surveys used in the validity testing have evidenced the face and external validity of the tool. The use of I.ROC as a valid measure of recovery within a Scottish mental health population is therefore supported.

\section{Limitations}

Although 171 participants were recruited for the current study, the robustness of the results would be improved by increasing sample size. For factor analysis, for example, it has been argued that the bigger the sample size, the better the results, with a minimum sample size of 300 recommended by some. ${ }^{52}$ Although others have argued that a sample size of between 100 and 200 may be sufficient, ${ }^{53}$ the general consensus remains 'the bigger the better'. 
Participants were drawn from Penumbra's existing service user base with testers made up of Penumbra staff. This presented a readily available sample group and minimised the likelihood of breaches of client confidentiality, but it should also be recognised that these choices may have positively influenced aspects of the results. Specifically, participants may have been more likely to favour the I.ROC over other instruments and staff may have, albeit inadvertently, reinforced this. The collection of data from individuals not connected with Penumbra using data collectors from outside the organisation would be useful in terms of determining the extent to which pre-existing familiarity with the tool may have affected the results.

The I.ROC has yet to be benchmarked against the most widely used measures of recovery in the UK, the Recovery Star and the QPR. As noted earlier, at the time of the present study there were no published data on the psychometric properties of the Recover Star. This has now changed. ${ }^{22,23}$ Although it is clear that further work needs to be done on the Recovery Star before it can be recommended as a routine clinical measure, as the current 'market leader' it may now be appropriate to examine similarities and differences between this tool and I.ROC. As QPR has also been used as a recovery outcomes measure within research in the UK, ${ }^{54}$ comparisons with this tool would also prove useful.

Although I.ROC has been used within Penumbra with the majority of their clients for a number of years, recent changes have been made to the questions in response to service user feedback at the beginning of this study. These data have been collected by staff with minimal training in research methods and without standardised instructions and it has not been used in the current analysis. As a result, neither test-retest nor interrater reliability was explored in the current study. Both are clearly vital in terms of further establishing the usefulness of I.ROC. Future work might usefully consider these as part on an ongoing project to examine the reliability, validity and usability of this tool.

Notwithstanding, this study provides strong preliminary data to support the use of I.ROC as a measure of recovery in mental health. Its brevity and clarity support its routine use by a broad spectrum of service users with mental health problems and by busy front-line mental health workers. Undoubtedly, further testing is required, yet I.ROC compares very well with existing measures.

Copies of the tool are available from Penumbra on request. Training is required for use of I.ROC.

\section{Funding}

The work was funded by a Knowledge Transfer Partnership, from the Technology Strategy Board and the Scottish Funding Council.

\section{About the authors}

Bridey Monger, Research and Information Officer, Penumbra, and School of Social and Health Sciences, University of Abertay Dundee; Scott M. Hardie CPsychol AFBPsS, Director of Research and Research Degrees, School of Social and Health Sciences, University of Abertay Dundee; Robin Ion, Division Leader - Nursing and Counselling, School of Social and Health Sciences, University of Abertay Dundee; Jane Cumming, Development Manager, and Nigel Henderson, Chief Executive, Penumbra, Edinburgh.

\section{References}

1 Anthony WA. Recovery from mental illness: the guiding vision of the mental health service system in the 1990s. Psychosoc Rehabil 1993; 16: $11-23$

2 Armstrong M. Veteran's perspective. Psychiatr Rehabil J 2010; 33: 260-1.

3 Piat M, Sabetti J, Couture A. What does recovery mean for me? Perspectives of Canadian mental health consumers. Psychiatr Rehabil J 2009; 32: 199-207.

4 Neil ST, Kilbride M, Pitt L, Nothard S, Welford M, Sellwood W, et al. The questionnaire about the process of recovery (QPR): a measurement tool developed in collaboration with service users. Psychosis 2009; 1: 1-11.

5 Bradstreet S, McBrierty R. Recovery in Scotland: beyond service development. Int Rev Psychiatry 2012; 24: 64-9.

6 Lloyd C, King R, Moore L. Subjective and objective indicators of recovery in severe mental illness: a cross-sectional study. Int J Soc Psychiatry 2010; 56: 220-9.

7 Clarke SP, Oades LG, Crowe TP, Caputi P, Deane FP. The role of symptom distress and goal attainment in promoting aspects of psychological recovery for consumers with enduring mental illness. J Ment Health 2009; 18: 389-97.

8 Tilley S, Cowan S. Recovery in mental health policy: good strategy or bad rhetoric? Crit Public Health 2011; 21: 95-104.

9 Davidson L, Roe D. Recovery from versus recovery in serious mental illness: one strategy for lessening confusion plaguing recovery. $J$ Ment Health 2007; 16: 459-70.

10 Trauer T. Outcome measurement in chronic mental illness. Int Rev Psychiatry 2010; 22: 99-113.

11 Davidson L, O'Connell MJ, Tondora J, Lawless M, Evans AC. Recovery in serious mental illness: a new wine or just a new bottle? Prof Psychol Res Pract 2005; 36: 480-7.

12 Stickley T, Wright $\mathrm{N}$. The British research evidence for recovery, papers published between 2006 and 2009 (inclusive). Part One: a review of the peer-reviewed literature using a systematic approach. J Psychiatr Ment Health Nurs 2011; 18: 247-56.

13 Stickley T, Wright $\mathrm{N}$. The British research evidence for recovery, papers published between 2006 and 2009 (inclusive). Part Two: a review of the grey literature including book chapters and policy documents. J Psychiatr Ment Health Nurs 2011; 18: 297-307.

14 Bonney S, Stickley T. Recovery and mental health: a review of the British Literature. J Psychiatr Ment Health Nurs 2008; 15: 140-53.

15 Trauer T, Callaly T, Herrman $\mathrm{H}$. Attitudes of mental health staff to routine outcome measurement. J Ment Health 2009; 18: 288-96.

16 Burgess P, Pirkis J, Coombs T, Rosen A. Review of Recovery Measures Version 1.0. Australian Mental Health Outcomes and Classification Network, 2010.

17 Fisher DG, Pilon D, Hershberger SL, Reynolds GL, LaMaster SC, Davis M. Psychometric properties of an assessment for mental health recovery programs. Community Ment Health J 2009; 45: 246-50.

18 Campbell-Orde T, Chamberlin J, Carpenter J, Leff HS (eds) Measuring the Promise: A Compendium of Recovery Measures, Vol. 2. Human Services Research Institute, 2005.

19 Mueser KT, Gingerich S, Salyers MP, McGuire AB, Reyes RU, Cunningham H. Illness Management and Recovery (IMR) Scales (Client and Clinician Versions). In Measuring the Promise: A Compendium of Recovery Measures, Vol. 2 (eds T Campbell-Orde, J Chamberlin, J Carpenter, HS Leff). Human Services Research Institute, 2005.

20 Smith-Merry J, Freeman R, Sturdy S. Implementing recovery: an analysis of the key technologies in Scotland. Int J Ment Health Syst 2011; 5: 1-12.

21 MacKeith J, Burns S. Mental Health Recovery Star. Mental Health Providers Forum and Triangle Consulting, 2008.

22 Dickens G, Weleminsky J, Onifade Y, Sugarman P. Recovery Star: validating user recovery. Psychiatrist 2012; 36: 45-50.

23 Killaspy H, White S, Taylor TL, King, M. Psychometric properties of the Mental Health Recovery Star. Br J Psychiatry 2012; 201: 65-70. 
24 Burns S, MacKeith J, Graham K. Using the Outcomes Star. Impact and Good Practice. Homeless Link, 2008.

25 Monger B, lon R, Henderson N, Cumming J, Hardie S. Outcome measurement in a Scottish mental health charity. Ment Health Today 2012; March/April: 24-7.

26 Giffort D, Schmook A, Woody C, Vollendorf C, Gervain M. Recovery Assessment Scale. Illinois Department of Mental Health, 1995.

27 Corrigan PW, Giffort D, Rashid F, Leary M, Okeke I. Recovery as a psychological construct. Community Ment Health J 1999; 35: 231-9.

28 Eisen SV. Behavior and Symptom Identification Scale (BASIS-32). In Outcomes Assessment in Clinical Practice (eds LI Sederer, B Dickey): 65-9. Williams \& Wilkins, 1996.

29 Eisen SV, Wilcox M, Leff HS, Schaefer E, Culhane MA. Assessing behavioural health outcomes in outpatient programs: reliability and validity of the BASIS-32. J Behav Health Serv Res 1999; 26: 5-17.

30 McNaught M, Caputi P, Oades LG, Deane FP. Testing the validity of the Recovery Assessment Scale using an Australian sample. Aust N Z J Psychiatry 2007; 41: 450-7.

31 Corrigan PW, Salzer M, Ralph RO, Sangster Y, Keck L. Examining the factor structure of the Recovery Assessment Scale. Schizophr Bull 2004; 30: $1035-41$

32 Andresen R, Caputi P, Oades L. Do clinical outcome measures assess consumer-defined recovery? Psychiatry Res 2010; 177: 309-17.

33 Andresen R, Caputi P, Oades L. Stages of recovery instrument: development of a measure of recovery from serious mental illness. Aust N Z J Psychiatry 2006; 40: 972-80.

34 Weeks G, Slade M, Hayward M. A UK validation of the Stages of Recovery Instrument. Int J Soc Psychiatry 2011; 57: 446-54.

35 Coombs T, Stapley K, Pirkis J. The multiple uses of routine mental health outcome measures in Australia and New Zealand: experiences from the field. Australas Psychiatry 2011; 19: 247-53.

36 Eisen SV, Dill DL, Grob MC. Reliability and validity of a brief patient report instrument for psychiatric outcome education. Hosp Community Psychiatry 1994; 45: 242-7.

37 Jerrell JM. Behaviour and Symptom Identification Scale 32. J Behav Health Serv Res 2005; 32: 341-6.

38 Song L, Hsu S. The development of the Stages of Recovery Scale for persons with persistent mental illness. Res Social Work Pract 2011; 21: 572-81.

39 The Colorado Health Networks Partnership. Consumer Recovery Outcomes System (CROS 3.0). In Measuring the Promise: A Compendium of Recovery Measures, Vol. 2 (eds T Campbell-Orde, J Chamberlin, J Carpenter, HS Leff). Human Services Research Institute, 2005.
40 Chiba R, Miyamoto $Y$, Kawakami N. Reliability and validity of the Japanese version of the Recovery Assessment Scale (RAS) for people with chronic mental illness: scale development. Int I Nurs Stud 2010; 47: 314-22.

41 Dunn EC, Rogers ES, Hutchinson DS, Lyass A, MacDonald Wilson KL, Wallace LR, et al. Results of an innovative university-based recovery education program for adults with psychiatric disabilities. Adm Policy Ment Health 2008; 35: 357-69.

42 Wong L, Harris M, Cotton S, Edwards J. Routine outcome assessment and feedback for clinicians: a pilot in an early psychosis service. J Ment Health 2006; 15: 279-88.

43 Russinova Z, Wewiorski NJ, Lyass A, Rogers ES, Massaro JM. Correlates of vocational recovery for persons with schizophrenia. Int Rev Psychiatry 2002; 14: 303-11.

44 Chen EYH, Tam DKP, Wong JWS, Law CW, Chiu CPY. Self-administered instrument to measure the patient's experience of recovery after firstepisode psychosis: development and validation of the Psychosis Recovery Inventory. Aust N Z J Psychiatry 2005; 39: 493-9.

45 Smith M, Smith P. Testing People at Work: Competencies in Psychometric Testing. BPS Blackwell, 2005.

46 Henson RK. Understanding internal consistency reliability estimates: a conceptual primer on coefficient alpha. Meas Eval Counsel Dev 2001; 34 $177-89$.

47 George D, Mallery P (eds) SPSS for Windows Step by Step: A Simple Guide and Reference. 11.0 update (4th edn). Allyn \& Bacon, 2003.

48 Gliem JA, Gliem RR. Calculating, Interpreting, and Reporting Cronbach's Alpha Reliability Coefficient for Likert-Type Scales. Midwest Research to Practice Conference in Adult, Continuing, and Community Education, 2003

49 Kaiser HF. An index of factorial simplicity. Psychometrika 1974; 39: 31-6.

50 Mooi E, Sarstedt MA. Factor analysis. In Concise Guide to Market Research: The Process, Data, and Methods using IBM SPSS Statistics. Springer-Verlag, 2011: 201-36.

51 Andresen R, Caputi $\mathrm{P}$, Oades L. Do clinical outcome measures assess consumer-defined recovery? Psychiatry Res 2010; 177: 309-17.

52 Comrey AL, Lee HB. A First Course in Factor Analysis. Psychology Press, 1992.

53 Matsunaga M. How to factor-analyze your data right: do's, don't's and how-to's. Int J Psychol Res 2010; 3: 97-110.

54 Slade $M$, Bird V, Le Boutillier CL, Williams J, McCrone P, Leamy M. REFOCUS Trial: protocol for a cluster randomised controlled trial of a pro-recovery intervention within community based mental health teams. BMC Psychiatry 2011; 11: 185. 